\title{
La réforme électrique française : une alternative concurrentielle à l'opérateur dominant ?
}

\author{
J-M Glachant, ADIS-GRJM, Université Paris-Sud, et M. Saguan, Supélec
}

\begin{abstract}
La réforme électrique française présente un cas assez particulier de création d'un marché "concurrentiel" autour d'un ancien monopole intégré qui garde la totalité de ses actifs industriels. Ce nouveau marché se développe donc comme une frange concurrentielle autour de l'ancien monopole. L'opérateur dominant, qui possède un parc de production essentiellement nucléaire et hydraulique, a été capable de soutenir toutes les pressions de la concurrence pendant la période initiale de baisse des prix, et sans avoir à abuser de son pouvoir de marché. En conséquence, l'évolution du marché français vers un système concurrentiel banalisé ne semble pas pouvoir s'effectuer rapidement ni de manière spontanée.
\end{abstract}

Index Terms - Réforme électrique, Concurrence, Monopole, Frange concurrentielle, Dual Fuel.

\section{INTRODUCTION}

$\mathrm{L}$ a réforme électrique française est un cas typique de réforme sans restructuration industrielle des opérateurs dominants. C'est aussi un cas assez rare de création d'un marché «concurrentiel » autour d'un monopole d'Etat qui garde la totalité de ses actifs industriels. De ce fait, la principale caractéristique de la réforme française est de développer une frange concurrentielle autour de l'ancien monopole [1], [2]

Ce genre particulier de réforme électrique pose nécessairement de nombreuses questions intéressantes. Quelle frange concurrentielle peut se construire autour d'un monopole sans avoir détruit ce monopole? Quels effets ce procédé de réforme peut-t-il exercer sur le marché où opère l'ancien monopoleur? Ce type de réforme est-il durable ou transitoire ? Verra-t-on s'ouvrir plus tard une fenêtre plus large de contestation du monopole, notamment lors de la reprise de l'investissement en production ?

Nous remercions de nombreux amis, notamment D. Finon, M. Massoni, J-P Bouttes, C. Gence-Creux, V. Pignon, M-P Bongrain, J-M Coulondre, Y. Perez, F. Lévêque, V. Thouvenin, D. Newbery, R. Green, Y. Smeers, P. Joskow, I. Perez-Arriaga, P. Ranci, G. Lundberg, R. Belmans et C. von Hirchhausen pour les discussions animées qui ont accompagné l'écriture de ce papier. Bien entendu aucun d'entre eux n'est responsable du résultat final.

J.M. Glachant est professeur d'économie à l'Université de Paris-Sud, ADIS-GRJM, Faculté Jean-Monnet, 54 bd. Desgranges, 92331 Sceaux Cedex, (email: jean-michel.glachant@jm.u-psud.fr; site web: www.grjm.net); M. Saguan est ingénieur et chercheur en génie électrique et économie à la fois à SUPELEC (Projet €nergie) et à l'ADIS-GRJM, Université de Paris Sud (email: marcelo.saguan@supelec.fr, site web www.supelec.fr/ecole/eei/energie/accueil.html).
Dans une première partie, nous chercherons si une concurrence étrangère, au moins potentielle, peut constituer une frange concurrentielle extérieure autour du monopole français. Ayant précisé les points forts et les points faibles de la concurrence extérieure vis-à-vis du monopole français, nous examinerons, dans une seconde partie, quelles dispositions concurrentielles ont été prises sur le marché national pour atténuer le pouvoir du monopole, à l'initiative d'autorités françaises ou européennes. Dans une troisième partie, nous chercherons si ces différentes mesures d'encerclement concurrentiel du monopole ont eu des effets visibles, en volume ou en prix, sur le marché national.

Dans une quatrième partie, nous examinerons le scénario d'une intensification de la contestation du monopole lors de la reprise de l'investissement, notamment lorsque le parc nucléaire français arrivera à l'échéance de son renouvellement. Ayant constaté qu’on ne prévoit pas de reprise massive de l'investissement en France au cours de la prochaine décennie, et que ses modalités pratiques demeurent très incertaines, nous nous demanderons, dans une cinquième et dernière partie, ce qui pourrait renforcer la dynamique concurrentielle dans le cadre actuel de vive remontée des prix de gros, même si les axiomes de la politique française ne changeaient pas (pas de restructuration industrielle en production ou de désinvestissement forcé du champion national).

\section{LE MONOPOLE FRANÇAIS PEUT-IL ETRE CONTESTE A PARTIR DE L'ETRANGER?}

Un monopole national peut être contesté à partir de l'étranger si son réseau de transport permet l'importation de la production des pays voisins, ou si ce monopole est lui-même exportateur dans ces pays. Cette concurrence étrangère est plus sérieuse dans un contexte de surcapacités de production, et quand les coûts de production de court terme sont favorables aux producteurs étrangers. Une partie seulement de toutes ces conditions spéciales s'appliquent au cas du monopole français de l'électricité. Mais elles suffisent à créer un cadre de concurrence extérieure potentielle.

Certes, dans le secteur électrique, EDF possède plus de 90 \% des capacités de production et $100 \%$ du réseau de transport. EDF exploite environ $95 \%$ des réseaux de distribution (mais ces réseaux appartiennent aux collectivités locales) et fournit environ $95 \%$ des clients non éligibles à la concurrence (les autres clients non éligibles relevant de distributeurs publics ou 
coopératifs non étatiques : les DNN) [3], [4]. EDF est ainsi la plus grande entreprise électrique d'Europe avec près de 500 TWh de production. En dehors d'EDF, il n'existe qu'environ 25 TWh de production «libre ", le reste de la production indépendante étant autoconsommée ou revendue à EDF dans le cadre d' "obligations d'achat» rattachées au "service public de la production » (notamment pour la cogénération et les renouvelables).

Le réseau de transport d'électricité est toujours une entité interne d'EDF, mais sa direction et son exploitation ont été séparées de la chaîne de commandement d'EDF et placées sous le contrôle direct du régulateur indépendant (CRE = Commission de régulation de l'Energie). Le régulateur surveille et garantit la séparation comptable et l'autonomie de comportement du transporteur (y compris pour son programme d'investissement, son financement et ses prix). En pratique, le réseau de transport français se conduit donc comme une entité largement autonome, et il n'y a pas de plaintes de grands consommateurs ou de concurrents d'EDF contestant l'impartialité du comportement du transporteur [5]. Du fait que le réseau de transport ne pratique pas de collusion avec le producteur dominant, le quasi-monopole EDF se trouve placé sur un réseau de transport ouvert par les directives européennes et n'est pas protégé des importations de production étrangère. Sur les quatre frontières françaises (Allemagne, Belgique, Angleterre et Espagne) où la capacité d'importation peut être facilement définie avec la méthodologie ETSO, cette capacité atteint 10350 MW garantis pour la période hivernale. Sur les deux frontières (Suisse et Italie) où la capacité maximale d'importation n'est pas définie avec la même précision, elle est cependant, du côté français, à peu près égale à la capacité d'exportation (5 350 MW), avec notamment plus de 2400 MW de capacité d'importation garantie en provenance de la Suisse seule [6]. La pointe de demande d'hiver en France étant d'environ 80 000 MW (y compris les exportations pour environ 13\%), la capacité totale d'importation garantie en France, avec plus de $14000 \mathrm{MW}$, était donc supérieure à $20 \%$ au $70 \mathrm{GW}$ de la demande intérieure moyenne. La taille du marché des clients éligibles atteignant plus du $1 / 3$ du marché intérieur français jusqu'en 2004, et plus des $2 / 3$ à partir du $1^{\mathrm{er}}$ juillet 2004, la capacité physique garantie d'importation en France était donc égale à plus de $50 \%$ du marché éligible avant 2004, puis à environ $30 \%$ depuis juillet 2004. Cette capacité physique d'importation en France est d'autant plus accessible à l'initiative étrangère qu'il n'existe aucune (ou presque aucune) congestion à l'importation vers la France. Enfin, la partie du marché français ouverte à la concurrence extérieure entre 1999 et 2004 était celle des clients industriels. Or ces clients représentent la demande la plus sensible au prix et la plus susceptible d'acheter à des producteurs étrangers.

TABLEAU I

SEUILS D'OUVERTURE DU MARCHE FRANÇAIS

Février 1999: 40 GWh (450 clients; 22 \% du marché)
Février 2000: 16 GWh (1 400 clients; $30 \%$ du marché)

Février 2003: 7 GWh (3 100 clients; $37 \%$ du marché)

Juillet 2004: tous les clients non domestiques (3 500000 clients; $68 \%$ du marché)

Juillet 2007: tous les clients domestiques (100\% du marché)

Par ailleurs, l'ancien monopole français est également soumis à la concurrence des producteurs étrangers sur tous les marchés où EDF exporte. Ces exportations sont considérables car EDF est le premier exportateur mondial avec plus de 70 TWh (environ $15 \%$ de sa production). Au total, du fait de son activité d'exportation et de l'ouverture de son réseau de transport, EDF est aujourd'hui un ancien monopole soumis à une concurrence extérieure potentielle sur un volume important de fourniture (au moins $150 \mathrm{TWh}$ ).

TABLEAU II

EXPORTATIONS FRANÇAISES D'ELECTRICITE EN 2001 (en GWh)

\begin{tabular}{|llll|}
\hline Belgique & Exports & Imports & Exports Nettes \\
Allemagne & 11651 & 204 & 11447 \\
Italie & 14924 & 542 & 14382 \\
Espagne & 18030 & 459 & 17571 \\
Suisse & 6768 & 1242 & 5526 \\
Angleterre & 9839 & 1816 & 8023 \\
Autres & 11522 & 208 & 11314 \\
Total & 127 & - & 127 \\
\hline
\end{tabular}

Cette situation de concurrence potentielle aux frontières du monopole français était renforcée par l'existence de surcapacités de production en France et dans la plupart des pays connectés au réseau français de transport, au moins jusqu'en 2003 ou 2004. Cinq des six pays frontaliers de la France présentaient encore à la pointe de l'hiver 2003-2004 des surcapacités « réelles » (centrales en état de marche) d’au moins $10 \%$ ou plus de $10 \%$ au dessus des réserves de $5 \%$ recommandées par l'UCTE [7].

Selon les études du transporteur français RTE publiées en 2004, la surcapacité à la pointe hivernale en Europe de l'Ouest continentale (évaluée en 2004 à 12 GW au dessus des $5 \%$ de réserves UCTE) pouvait se maintenir jusqu'en 2008 (où elle baisserait à 7 GW) avant de disparaître en 2009 [8].

Cependant, même en période de surcapacités, la concurrence potentielle des producteurs étrangers n'est pas menaçante pour la production française (qui relève des filières nucléaire ou hydraulique pour 90 à $95 \%$ de ses volumes). Certes, avec près de 80 \% d'électricité d'origine nucléaire, le parc nucléaire français n'atteint pas des records de productivité de ses centrales (moins de 7000 heures à pleine puissance par an en moyenne). Mais ceci ne modifie pas substantiellement l'équation de ses coûts de court terme (Fuel + Operation \& Maintenance). Le nucléaire français, secondé par un bon parc hydraulique, peut donc faire face aisément à toute « guerre de prix » qui serait lancée par les centrales thermiques classiques des producteurs étrangers, notamment britanniques, allemands ou espagnols. Même en Grande-Bretagne, en mai 2004, John Bower estimait les coûts de court terme du nucléaire ancien à seulement les 2/3 des coûts de court terme des centrales charbon et gaz CCGT en fonctionnement. Seul l'hydraulique 
suisse pourrait facilement percer le marché français en termes de coût de court terme, mais il préfère bien sûr se déverser là où les prix sont plus rémunérateurs qu'en France (comme en Italie).

En conclusion, il existe bien une concurrence extérieure potentielle aux frontières de l'ancien monopole français, soit parce que celui-ci est fortement exportateur dans les pays frontaliers (plus de $70 \mathrm{TWh}$ ), soit parce que son réseau de transport est capable d'importer en France une part importante du marché français des consommateurs éligibles (du 1/3 à la $1 / 2$ ). Mais cette concurrence reste surtout potentielle, malgré les surcapacités de production en place en 2000-2004, car les coûts de court terme du nucléaire français ne peuvent pas être sérieusement concurrencés par les coûts de court terme du thermique classique des producteurs étrangers. Il est cependant arrivé, à certaines heures creuses de la nuit, que les centrales allemandes au lignite puissent offrir des prix inférieurs à ceux du nucléaire français.

\section{DES ATTENUATIONS DU POUVOIR DE L'ANCIEN MONOPOLE}

Puisque la concurrence extérieure reste surtout potentielle aux frontières du marché intérieur français, plusieurs dispositions concurrentielles ont été prises pour atténuer le pouvoir du monopole national, que ce soit par action directe sur l'offre ou la demande (assets sell off ; electricity release ; procurement auctionning; etc.), ou par action sur les mécanismes de marché ou sur l'accès au marché (power exchange ; balancing mechanism ; transmission capacity auction ; etc.).

Certes la présence d'un monopole industriel vertical et horizontal ne laisse pas beaucoup de place libre pour l'introduction de la concurrence. Mais il restait en France quelques marges disponibles en dehors d'EDF. Ces marges ont été agrandies par des actions réglementaires (soit du régulateur français, soit de la Commission européenne) ou par des initiatives commerciales (comme la création de la bourse Powernext).

TABLEAU III

PARTS DE MARCHE DES PRODUCTEURS EN 2002

\begin{tabular}{|lll|}
\hline Entreprise & Parts & $\begin{array}{l}\text { Technologies } \\
\text { Nucléaire, Hydro, Charbon, Turbines } \\
\text { EDF }\end{array}$ \\
autol
\end{tabular}

Quelques sources de «production indépendante » ont été progressivement séparées d'EDF et associées à des opérateurs étrangers (Electrabel, Endesa). Ce sont la CNR (Hydro, 16 TWh), la SNET (Charbon, 8 TWh) et la SHEM (Hydro, 2 TWh). En 2004, Electrabel, qui produit principalement en Belgique (où la frontière offre un capacité garantie d'importation en France de près de $3000 \mathrm{MW}$ ), a pu ajouter à ses participations dans l'hydraulique français (CNR et SHEM) un accord d'exploitation de centrales communes avec EDF lui permettant de disposer de près de $1000 \mathrm{MW}$ de nucléaire en France. Electrabel annonce donc un objectif de pénétration de $10 \%$ du marché français éligible (soit 30 TWh d'ici 2007).

D'autre part, toujours du côté de l'offre, l'autorité de concurrence de la Commission européenne (la DG COMP) a exigé d'EDF, en contrepartie de son entrée au capital de la compagnie allemande ENBW, la réalisation d'un electricity release, qui a pris le nom de VPP (Virtual Power Plants). Ces VPP, d'un montant total de $6000 \mathrm{MW}$, sont entrés en exercice en Janvier 2002. Elles devaient durer au moins jusqu'à la fin de 2006. Mais EDF a devancé les souhaits de la Commission européenne en les laissant se prolonger au delà au vu de la situation concurrentielle sur le marché français. Ces VPP sont structurés autour d'enchères de 3 produits : VPP Base Load (8 Euros par MWh soutiré + prime fixe offerte aux enchères) ; VPP Peak (23 à 26 Euros par MWh soutiré + prime fixe offerte aux enchères); et $P P A$ (fourniture en base du $1^{\mathrm{er}}$ novembre au 31mars au prix offert aux enchères). Les durées de vie de ces produits sont variables (de 3 mois à 3 ans), mais la durée annuelle est la plus courante (avec $2500 \mathrm{MW}$ vendus). Depuis l'intervention du régulateur en juillet 2002, c'est la bourse française Powernext qui réalise directement auprès du transporteur RTE les nominations journalières des VPP par leurs propriétaires. EDF Producteur n'étant informé que du volume total de toutes ces nominations journalières.

Du côté de la demande, le régulateur français a amplifié l'ouverture concurrentielle du marché des éligibles en demandant au transporteur RTE de couvrir son approvisionnement des pertes du réseau (13 TWh) par un mécanisme d'enchères ouvert à tous (producteurs ou traders). D'autre part, en février 2003, une centaine de distributeurs français indépendants d'EDF (les DNN) sont devenus clients éligibles pour leurs propres achats en gros d'énergie (environ 1 TWh au total).

En dehors de ces deux nouveautés, toutes les autres modifications concurrentielles majeures de la demande viennent du calendrier législatif d'ouverture du marché éligible (22\% du marché national en $1999=90$ TWh ; $30 \%$ en $2000 ; 37 \%$ en 2003 ; et $68 \%$ en $2004=290$ TWh).

Outre ces actions directes sur l'offre ou la demande, d'autres actions ont aménagé ou consolidé des mécanismes concurrentiels de marché ou d'accès aux marchés. La plus importante de ces actions, lors du démarrage de la réforme, a été la neutralisation sous la tutelle du régulateur français du comportement du transporteur RTE, demeuré une entité interne d'EDF. Le régulateur français, créé par la loi de février 2000, n'a pas obtenu des pouvoirs complets sur le transporteur. Ainsi les tarifs de transport proposés par le régulateur peuvent être refusés par le ministre mais non modifiés par lui (il s'en est suivi une "guerre froide » des tarifs qui a duré de l'année 2000 jusqu'à juillet 2002 !). Mais les pouvoirs du régulateur ont été suffisants pour assurer une réelle autonomie du transporteur, avec le soutien actif des 
dirigeants du transport (le directeur du transport n'étant pas nommé par le président d'EDF et n'ayant pas à lui obéir).

Comme la France a peu de congestions aux frontières pour l'importation et peu de congestions internes, l'accès au réseau pour les producteurs étrangers ne présente pas à priori de grosses difficultés. D’autant moins que le tarif français du transport couvre toutes les charges du réseau et du système (coûts fixes, pertes, services auxiliaires et congestions internes) avec un seul « Timbre Poste » payé entièrement par les consommateurs ( $G=0 ; \mathrm{L}=100 \%)$. Après l'accord européen sur le paiement d'une charge d'accès transfrontalier (cross border fee), le régulateur français a incorporé cette charge au Timbre Poste du tarif d'accès au réseau. Pour les producteurs étrangers, l'accès au réseau de transport pour importation en France est donc à peu près libre et gratuit (hors règlement des écarts = imbalances).

Le règlement des écarts (imbalances settlement) a d'abord été opéré dans le cadre d'un contrat de fourniture quasi exclusive par EDF à un prix tarifé (par exemple, en été : écarts négatifs à $23-26$ euros et écarts positifs à 8 Euros). Le régulateur a ensuite demandé la transformation de ce procédé en un mécanisme d'ajustement ouvert à toutes les offres d'ajustement (producteurs indépendants et cogénérateurs, producteurs étrangers, consommateurs). Le nouveau mécanisme est plus concurrentiel que l'ancien dispositif français, bien qu'il ne crée pas non plus un marché de court terme de l'énergie [9]. Il fonctionne, depuis avril 2003, avec deux prix des écarts (un à la hausse et un à la baisse) qui comprennent chacun un élément de pénalité de $20 \%$ en plus du coût moyen d'achat supporté par le transporteur. En ce qui concerne la valorisation des écarts favorables au système électrique, le mécanisme français les rémunère au prix en J-1 (Day Ahead) de la bourse d'électricité Powernext.

Cette bourse d'électricité française a été créée en 2001 par le marché financier franco-belgo-néerlandais Euronext (1/3 du capital) en liaison avec un consortium de trois transporteurs (français RTE, belge Elia et néerlandais Tennet ; $18 \%$ du capital), et la participation de cinq énergéticiens européens dont les électriciens EDF, Electrabel et Endesa (20\% à eux trois). Exclusivement J-1 (Day Ahead) pendant deux ans et demi, Powernext a ouvert en juin 2004 un marché de futures traitant des contrats au mois, au trimestre ou à l'année (jusqu'à deux ans). Les liens entre cette bourse et le transporteur français d'électricité sont étroits, et le président du conseil d'administration de Powernext n'est autre que le directeur du "système électrique » du transporteur RTE. Le transporteur français garantit notamment l'acheminement sur son réseau de toutes les transactions Day Ahead et Futures opérées sur la bourse. D'autres liens techniques et commerciaux rapprochent le transporteur et la bourse, que ce soit pour les nominations des VPP, les nominations d'autres échanges entre opérateurs en vue du règlement des écarts, ou pour la valorisation par le transporteur des écarts favorables au système. Tous ces rapprochements ont été soutenus par le régulateur français. D'autre part, le transporteur gère également un mécanisme d'enregistrement («nomination ») de transfert de «blocs » en
J-1 entre opérateurs du marché bilatéral (ou marché OTC = Over The Counter $=$ «Sous le comptoir »). A partir de la fin 2002, à la demande du régulateur, une demi douzaine de fenêtres infra journalières (infraday) de transfert de «blocs " entre opérateurs du marché OTC ont été ouvertes par le transporteur.

Enfin, des mécanismes concurrentiels d'allocation des capacités de transport ont été mis en place sur la ligne entre la France et l'Angleterre. Ils couvrent toutes les échéances, de l'infra journalier à l'année. Par ailleurs, les transporteurs français et anglais peuvent aussi s'échanger leurs réserves sur cette même ligne, et ils travaillent pour l'ouvrir à des offres d'ajustement réciproques sur leurs mécanismes nationaux d'ajustement. Aucune autre frontière française ne connaît de régime plus concurrentiel. Sur les autres frontières, le transporteur français a d'abord pratiqué un système dit de "priorité » pour les exportations sortant de France (les opérateurs qui utilisaient à moins de $75 \%$ les capacités retenues perdaient leur priorité en cas de congestion). Avec l'Italie, un système de prorata attribuait des quotas de capacité d'interconnexion aux clients éligibles italiens sur la base de leur consommation antérieure. A partir de 2005, et en 2006, des mécanismes de marché d'allocation des interconnections ont été progressivement mis en place sur la plupart des frontières. Cependant, aux frontières espagnole et italienne, des difficultés de coopération avec le transporteur (GRTN) ou le PX national (= le marché espagnol OMEL) rendent toujours difficile la refonte des régimes d'accès. Sur la frontière belge et jusqu'aux Pays-Bas, les transporteurs, les PXs et les régulateurs des trois pays se sont engagés dans la construction d'une interface intégrée d'accès aux trois marchés appelée «Market Coupling » [10].

Toutes ces dispositions prises pour construire autour du monopole français un « cercle concurrentiel » peuvent faciliter l'exercice de la concurrence extérieure sur le marché français (notamment l'accès au réseau de transport et l'usage des capacités d'interconnexion, l'ouverture d'une bourse et d'un nouveau mécanisme d'ajustement). Mais ces dispositions ne peuvent pas augmenter le potentiel concurrentiel des opérateurs étrangers en France même. Seules les cessions d'actifs de production (CNR, SHEM et SNET) et les VPP ont $\mathrm{pu}$ apporter ce supplément d'offre concurrentielle pour stimuler le marché national. Mais alors quels résultats a-t-on pu obtenir avec un tel procédé de réforme ?

\section{DES EFFETS CONCURRENTIELS SUR LES VOLUMES ET LES PRIX SUR LE MARCHE INTERIEUR FRANÇAIS ?}

Après plusieurs années de réforme en France, on devrait pouvoir constater quels effets concurrentiels ont opéré sur les volumes et sur les prix. Les résultats montreront notamment si la frange concurrentielle s'est acclimatée en France, et quels effets elle a pu exercer sur le cœur «en monopole » du marché français. 


\section{A. L'évolution des volumes}

On peut évaluer la réforme électrique française à partir de plusieurs de ses effets volume. On s'intéressera tout d'abord à l'origine des ressources électriques détenues par les concurrents d'EDF (production indépendante? Importations? VPP ?), puis à l'utilisation que ces concurrents en font (vente aux clients éligibles ou au réseau de transport? Réexportation? $\mathrm{Ou}$... revente à EDF ?). On examinera ensuite l'évolution des parts de marché d'EDF et de ses concurrents sur le marché des clients éligibles. On finira par les mesures de concentration sur les différents compartiments du marché français et de la frange concurrentielle en France.

TABLEAU IV

IMPORTATIONS ET EXPORTATIONS D'ELECTRICITE EN FRANCE DE 1999 A 2003

\begin{tabular}{|llll|}
\hline & Imports & Exports & Exports nettes \\
Année 1999 & 5 & 68 & 63 \\
Année 2000 & 3,3 & 73 & 70 \\
Année 2001 & 4,2 & 73 & 69 \\
Année 2002 & 3,8 & 81 & 77 \\
Année 2003 & 7 & 73 & 66 \\
$\mathbf{2 0 0 3 / 2 0 0 2 ( \% )}$ & $\mathbf{+ 9 0} \%$ & $\mathbf{- 1 0 \%}$ & $\mathbf{- 1 4 \%}$ \\
\hline
\end{tabular}

(en TWh)

Le rôle relatif de la production indépendante, des importations et des VPP dans l'approvisionnement de l'offre concurrente d'EDF peut être précisé grâce aux données publiées par le transporteur ou par le régulateur français.

Après l'ouverture du marché français, entre 1999 et 2003, la concurrence étrangère potentielle n'a suscité aucune hausse globale des importations en France, ni aucune diminution importante des exportations. Pendant ces premières années, les importations se sont tenues entre $0,75 \%$ et $1,5 \%$ d'une consommation intérieure évoluant entre 431 TWh (en 1999) et 467 TWh (en 2003), et le solde exportateur français des années 2000-2002 a atteint un record historique. On constate cependant une évolution significative des importations et des exportations à partir de 2003. Mais elle comportait des effets climatiques exceptionnels qui pouvaient ne pas se reproduire. En fait, 2005 a aussi été une année record d'importation avec environ 30TWh bruts (contre 90 TWh bruts d'export) [3].

Les données du régulateur sont plus détaillées [5]. Elles montrent que les ressources utilisées par l'ensemble des concurrents d'EDF sur le territoire français ont évolué d'un total de $1800 \mathrm{GWh} /$ mois en septembre 2001 à $7200 \mathrm{GWh} /$ mois en mars 2004 (+ 400\% d'augmentation).

En comparant des mois identiques, on constate qu'entre mars 2003 et mars 2004 la production indépendante n’a pas contribué significativement à cette augmentation en volume des ressources, tandis que les importations ont apporté un supplément de +500 GWh à +1000 GWh / mensuels. Ainsi, au premier trimestre 2004, les importations par les concurrents d'EDF ont atteint le plus haut niveau observé sur les trois dernières années. Cependant, l'essentiel de leurs ressources viennent toujours des VPP qui apportent près de $4000 \mathrm{GWh}$ en mars 2004 (environ $55 \%$ du total des ressources).

L'utilisation de ces ressources par les concurrents d'EDF a aussi connu plusieurs inflexions notables. Tout d'abord, c'est seulement en février 2003 que les ventes (directes ou indirectes) aux consommateurs éligibles ont dépassé en importance les ventes au transporteur RTE (pour les pertes du réseau) et les réexportations dans les pays étrangers. En mars 2004, ces ventes aux consommateurs éligibles ont atteint un record mensuel de $3000 \mathrm{GWh}$. Cependant, dès octobre 2003, le total des ventes pour les pertes de RTE et les réexportations a rattrapé en importance les ventes aux consommateurs éligibles, et a atteint lui -aussi en janvier 2004 et mars 2004 un record de $3000 \mathrm{GWh}$ mensuels. D'autre part, à partir de juillet 2003, le total des ventes des concurrents d'EDF est devenu sensiblement inférieur au total des ressources qu'ils collectent, avec un écart mensuel entre 500 GWh et 1200 GWh.

TABLEAU V

ACTIVITES DES CONCURRENTS D'EDF VUES PAR LE RESEAU DE TRANSPORT $\left(1^{\mathrm{ER}}\right.$ TRIMESTRE 2004)

\begin{tabular}{|l|l|l|l|l|l|}
\hline $\begin{array}{l}\text { (vendu } \\
\text { à...) }\end{array}$ & $\begin{array}{l}\text { Consommateurs } \\
\text { éligibles }\end{array}$ & $\begin{array}{l}\text { Pertes du } \\
\text { Transport }\end{array}$ & Exports & EDF & Total \\
\hline en GWh & 8,200 & 3,500 & 5,900 & 2,700 & 20,300 \\
\hline en $\%$ & $41 \%$ & $17 \%$ & $29 \%$ & $13 \%$ & $100 \%$ \\
\hline
\end{tabular}

TABLEAU VI

RESSOURCES DES CONCURRENTS D'EDF VUES PAR LE RESEAU DE TRANSPORT (1 ${ }^{\mathrm{ER}}$ TRIMESTRE 2004)

\begin{tabular}{|l|l|l|l|l|l|}
\hline $\begin{array}{l}\text { (coming } \\
\text { from }>\text { >) }\end{array}$ & $\begin{array}{l}\text { Independent } \\
\text { Generation }\end{array}$ & VPP & Imports & $/ /$ & Total \\
\hline in TWh & 3,900 & 11,400 & 5,000 & $/ /$ & 20,300 \\
\hline in $\%$ & $19 \%$ & $56 \%$ & $25 \%$ & $/ /$ & $100 \%$ \\
\hline
\end{tabular}

Cet écart montre que les concurrents d'EDF ne parvenaient pas à vendre toutes leurs ressources à la demande intérieure française ou à l'exportation, et qu'ils devaient finalement revendre tout leur excédent à EDF. Si cette tendance du $2^{\mathrm{e}}$ semestre 2003 et du premier trimestre 2004 était confirmée, il faudrait s'interroger sur l'efficacité des VPP pour construire une offre concurrentielle alternative sur le marché français. En mars 2004, l'activité de revente à EDF aurait atteint 1200 GWh, soit plus de 30\% des ressources VPP du mois. Enfin, la dernière des grandes modifications du premier trimestre 2004 est que les importations réalisées par les concurrents d'EDF ont atteint le niveau record de 5 TWh trimestriels, constituant ainsi $25 \%$ de leurs ressources totales.

Un autre indicateur en volume caractérisant la réforme électrique française est l'évolution des ventes sur le marché des clients éligibles. On le connaît sous le nom de «taux de switching».

En dehors des enchères pour les pertes du réseau, la percée des concurrents d'EDF a commencé à l'automne 2001 en frôlant les 10 \% du marché, puis a fait un nouveau bond au printemps 2002 en atteignant $16 \%$ en volume. Ce niveau n'a plus guère été dépassé pendant les douze mois suivants (avril 2002-mars 2003). Un nouveau palier a été atteint au deuxième trimestre 2003 avec une part de marché frôlant les 19 \% puis déclinant jusqu'à $16 \%$ en janvier 2004. Avec l'ouverture du marché éligible à tous les consommateurs non domestiques en juillet 2004, cette part des concurrents d'EDF a chuté puisque la taille du marché éligible est passée de 173 TWh à 318 
TWh. Les premières informations non officielles disponibles en France à la fin de 2004 montraient que quelques dizaines de milliers seulement des 4 millions de nouveaux consommateurs éligibles avaient effectivement changé de fournisseur.

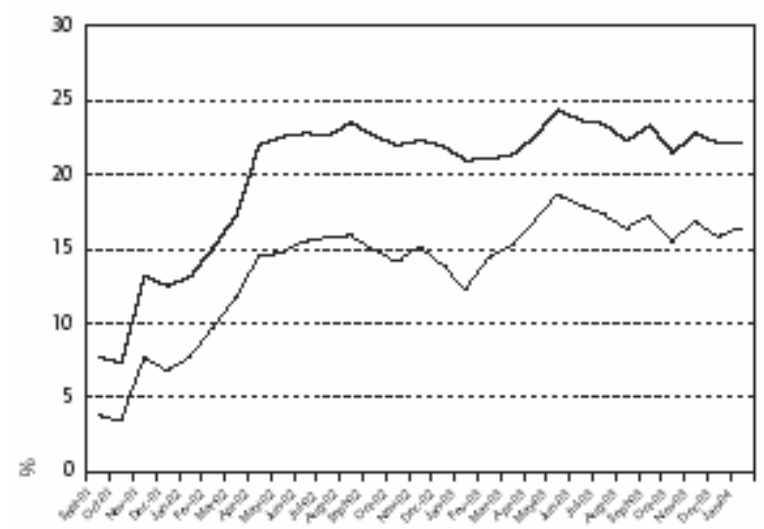

Fig. 1. Parts de marché en \% gagnées par les nouveaux entrants en France de septembre 2001 à janvier 2004, avec ou sans l'achat des pertes du réseau de transport, selon l'Agence Internationale de l'Energie en Juin 2004.

Les derniers indicateurs en volume capable de caractériser l'évolution du marché français et de sa frange concurrentielle sont ceux de la concentration.

Les indices de concentration des ventes établis par le régulateur français [5] suggèrent qu'EDF n'a pas d'activité nette d'alimentation de la bourse Powernext, ni de fourniture de pertes au transporteur, ni de vente de blocs aux responsables d'équilibres (balancing entities) sur le marché OTC. Et que ces trois activités ne sont pas non plus très concentrées entre les concurrents d'EDF (HHI inférieur à 800 hors EDF). Par contre, sur le marché des éligibles, les parts de marché d'EDF se situent toujours au dessus de 80 \% (mais en dessous de $83 \%$ ), et sur celui des exportations autour de 79 $\%$. Les concurrents d'EDF sont assez concentrés sur leur propre part du marché des éligibles hors EDF (HHI de 1618 hors EDF ; avec $90 \%$ des ventes aux cinq premiers et environ $35 \%$ au premier -Electrabel ?). Mais ils restent peu concentrés pour leurs activités d'exportation (HHI de 565 hors EDF).

Les indices de concentration des ressources du régulateur français [5] montrent qu'EDF contrôle environ 95 \% de la production (avec les obligations d'achat), mais moins de $40 \%$ des importations en France. Les concurrents d'EDF sont très concentrés dans leurs activités de production (HHI de 4617 hors EDF), et très peu concentrés pour toutes les autres ressources (HHI de moins de 750 pour les achats sur la bourse Powernext ou aux enchères VPP, pour les importations et pour les achats de blocs sur le marché OTC).

Le rapprochement des deux séries d'indices de concentration, indices des ventes et indices des ressources, suggère que la concurrence exercée vis à vis d'EDF sur le marché des éligibles est d'avantage structurée par la production indépendante en France que par les enchères de VPP ou par les importations. Les ressources des VPP et des importations restent dispersées entre de nombreux petits concurrents d'EDF. Ce qui correspond assez logiquement à la dispersion des leurs ventes sur la bourse Powernext, sur le marché des pertes pour le transporteur, sur les ventes de blocs en OTC ou sur les exportations.

\section{B. L'évolution des prix}

Ayant constaté que la concurrence étrangère demeurait surtout potentielle, et que $75 \%$ des ressources des concurrents d'EDF provenaient de la production en France (production indépendante ou VPP), nous pouvons maintenant nous intéresser aux effets prix. Dans le contexte français, caractérisé par un réseau de transport ouvert et fortement interconnecté, avec des surcapacités de production à l'intérieur et aux frontières, mais avec un avantage de coût en faveur de la production nationale, un monopoleur rationnel peut avoir deux comportements différents. Il peut, pour maximiser ses propres bénéfices, laisser la concurrence étrangère fixer le niveau des prix sur le marché de gros et le niveau des prix aux clients éligibles. Mais il peut aussi, alternativement, fixer des prix aux clients éligibles inférieurs à ses concurrents étrangers pour déprimer la rentabilité de leur entrée. Parallèlement, ce monopoleur peut garder en toute tranquillité une marge plus substantielle sur le segment des clients captifs, opérant ainsi des subventions croisées entre les deux segments, éligible et captif, de sa clientèle. Ces deux scénarios simples de "monopoleur raisonné par l'ouverture des frontières » peuvent être confrontés aux données de prix. Entre l'automne 2001 et le printemps 2004, les prix de gros français en base (baseload) semblent alignés sur les prix allemands, et leur sont parfois légèrement inférieurs, comme si les centrales françaises avaient des coûts de court terme très proches de ceux des centrales allemandes [11]. Ce résultat est tout à fait remarquable car, dans un scénario concurrentiel avec surcapacités de production, on s'attendrait à ce que les prix français en base se rapprochent plus des coûts de court terme du nucléaire français que des coûts de court terme du thermique allemand.

Les prix de gros à terme à l'échéance d'un an montrent aussi un fort parallélisme de niveau et d'évolution entre les prix français et les prix allemands, avec un léger retrait des prix français, sur toute la période mesurée (de 2001 au premier semestre 2004) [7] et [11].

S'agissant maintenant des prix finals, si on peut faire confiance aux statistiques de prix pour les gros consommateurs industriels, les prix français sont toujours parmi les deux plus bas entre 1999 et 2003 (inférieurs ou égaux à 50 Euros le MWh, dont environ 10 Euros de transport) et sont les plus bas trois années sur cinq. Sauf l'année 2000, les prix français sont inférieurs aux prix allemands (de 4 euros par MWh en 2001 et 2002 ; de 19 euros en 2003). Ceci suggère que le monopole français n'exploite pas à la hausse toute sa marge d'action en prix sur le marché national des grands clients éligibles. 


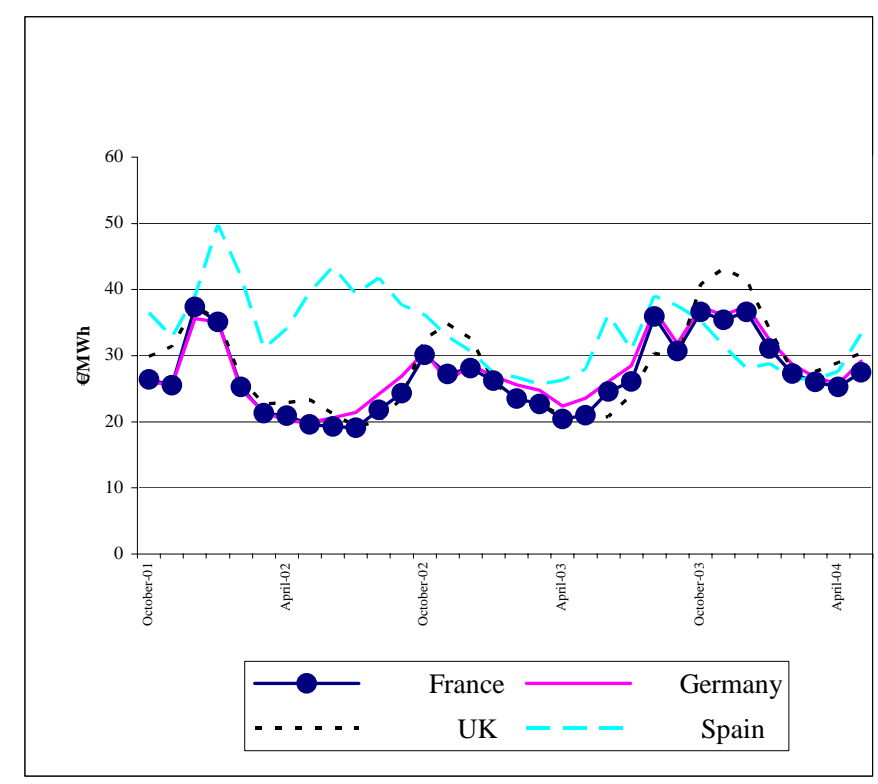

Fig. 2. Prix moyen mensuel en base sur les marchés OTC en France, Allemagne, Royaume-Uni et Espagne d'octobre 2001 à avril 2004 (en Euros / MWh).

Sur le marché des clients domestiques (non éligibles en France jusqu'en juillet 2007), c'est toujours le gouvernement français (propriétaire du monopole national) qui fixe les prix, et non le marché ou le régulateur indépendant. Le gouvernement français aurait pu souhaiter suivre les tendances baissières du marché de gros, et celles du marché des grands consommateurs industriels. Il pourrait, au contraire, préférer préserver une marge plus substantielle au fournisseur en monopole dans la fourniture des consommateurs non éligibles.

TABLEAU VII

MARGE PRIX DOMESTIQUES $3500 \mathrm{KWH}$ / PRIX GRANDS INDUSTRIELS 24 GWH (DONNEES EUROPEENNES D'EUROSTAT ; EN EUROS / MWH)

\begin{tabular}{|l|r|r|r|r|r|r|r|}
\hline & 1997 & 1998 & 1999 & 2000 & 2001 & 2002 & 2003 \\
\hline ITA & 108 & 108 & 104 & 90 & 78 & 68 & 70 \\
\hline ALL & 59 & 60 & 65 & 69 & 69 & 73 & 65 \\
\hline BEL & 61 & 63 & 63 & 62 & 61 & 56 & 56 \\
\hline G-B & 48 & 51 & 43 & 45 & 45 & 50 & 52 \\
\hline FRA & 46 & 44 & 45 & 44 & 43 & 43 & 44 \\
\hline ESP & 46 & 43 & 40 & 36 & 37 & 39 & 39 \\
\hline
\end{tabular}

Une mesure approximative de cette marge est donnée par la différence entre les prix des grands consommateurs industriels (à priori fixés par un marché concurrentiel) et ceux des consommateurs domestiques. Par rapport aux pays ayant les prix les plus fréquemment bas, la Grande-Bretagne et l'Espagne, la marge en France apparaît très raisonnable. Il n'y a donc pas de signe visible, en France, d'une politique prononcée de subventions croisées entre les clients encore en monopole et les clients éligibles.

Enfin, au-delà du seul niveau des prix en France, on peut s'interroger sur l'existence de liaisons concurrentielles sur le marché intérieur français entre les effets prix et les effets volume; notamment sur la capacité du marché français à réagir en volume, et à court terme, aux différentiels de prix de gros avec les marchés environnants. Cette donnée est particulièrement intéressante sur la liaison avec l'Angleterre qui est gérée par le dispositif le plus concurrentiel qui soit pratiqué en France sur les interconnexions. On constate bien l'existence de tels effets concurrentiels de court terme, avec des flux et des contre flux qui atteignent jusqu'à 12 ou 37 GWh par jour (soit 6 heures à 19 heures d'utilisation quotidienne de cette liaison de $2 \mathrm{GW}$ de capacité) [5]. Cumulés sur l'ensemble de l'année 2003, ces effets de variation des volumes entre la France et l'Angleterre ont même été assez remarquables. La réduction des exportations françaises a atteint - 5,6 TWh sur l'année tandis que l'augmentation des importations en provenance de l'Angleterre était de $+3,4 \mathrm{TWh}$, soit une évolution totale de 9 TWh annuels du solde des échanges (équivalent à 4500 Heures de pleine capacité de cette interconnexion sur une seule direction).

\section{UNE PLUS FORTE CONTESTATION DU MONOPOLE LORS DE LA REPRISE MASSIVE DE L'INVESTISSEMENT EN PRIODUCTION?}

Puisque la concurrence étrangère reste surtout potentielle, et que la frange concurrentielle intérieure n'a pas non plus pénétré le cœur du marché français, nous devons nous demander si ce n'est pas la reprise de l'investissement productif en France qui ouvrirait la contestation la plus forte de l'ancien monopole national. Ce scénario de « contestation par l'investissement» comprend de nombreux aspects que nous ne pourrons tous discuter. Nous chercherons d'abord quand pourrait avoir lieu une reprise massive de l'investissement et pour quel volume. Puis nous nous demanderons qui pourrait investir, pour quelle technologie, et à quel endroit.

La reprise massive de l'investissement peut avoir deux causes : le remplacement «normal » de centrales arrivées en fin de durée de vie, ou le remplacement accéléré des centrales existantes en raison de la percée d'une nouvelle technologie particulièrement compétitive. Nous avons déjà vu que le nucléaire français conservait un net avantage de coût vis-à-vis de toutes les autres technologies réalisables en France. Il ne reste donc qu'à examiner les scénarios d'évolution de la demande et du parc nucléaire, tels que les présentent les prévisions du transporteur français et les statistiques du parc nucléaire. RTE voit les surcapacités de l'Europe continentale de l'Ouest se maintenir encore quelques années, peut être jusqu’à 2008.

Pour l'évolution de la demande en France, RTE a construit 3 scénarios d'évolution dans son bilan prévisionnel pour 20062015.

TABLEAU VIII

TAUX DE CROISSANCE ANNUELLE DE LA CONSOMMATION D'ELECTRICITE EN FRANCE (EN \%) 


\begin{tabular}{|l|c|l|l|}
\hline Jusqu'en & 2010 & $2010-2015$ & $2015-2020$ \\
\hline Scenario R1 & 1.4 & 0.9 & 0.6 \\
\hline Scenario R2 & 1.3 & 0.9 & 0.5 \\
\hline Scenario R3 & 1.1 & 0.6 & 0.3 \\
\hline
\end{tabular}

TABLEAU IX

CONSOMMATION D'ELECTRICITE EN FRANCE (EN TWH)

\begin{tabular}{|l|c|l|l|}
\hline Année & 2010 & 2015 & 2020 \\
\hline Scenario R1 & 520 & 544 & 561 \\
\hline Scenario R2 & 513 & 536 & 550 \\
\hline Scenario R3 & 503 & 519 & 527 \\
\hline
\end{tabular}

De ces scénarios, le Transporteur français déduit que, même avec de faibles investissements productifs, le risque de défaillance pour l'année 2006 n'atteint que 1\% à 3\% pour une défaillance de 1 heure; mais, pour l'année 2010, 22\% de risque pour une défaillance de 11 heures (1.4 à 4 GW d'investissement seront donc nécessaires avant cette date); et enfin, en 2015, $83 \%$ de risque pour une défaillance de 87 heures (un supplément de 5GW sera donc nécessaire entre 2010 et 2015).

Cependant, plusieurs hypothèses additionnelles du TSO laissent penser qu'il n'y aurait pas beaucoup de place libre pour des investissements importants avant 2015. D'une part, des réductions volontaires de la pointe hivernale pourraient être achetées aux consommateurs (il y avait avant la réforme électrique $3 \mathrm{GW}$ de plus de réduction volontaire de la pointe). D'autre part, les exportations d'EDF pourraient reculer à mesure que des investissements nouveaux auraient lieu à l'étranger, dans le cadre d'une uniformisation progressive des coûts de production des centrales marginales sur toute l'Europe continentale. EDF pourrait aussi rééquiper de vieilles centrales thermiques gardées sous cocon (moothballed). Ensuite, aux USA, des industriels du nucléaire ont réussi à augmenter la productivité de réacteurs semblables aux réacteurs français de $5 \%$, ce qui créerait en France une capacité supplémentaire égale à deux à trois réacteurs (3000 MW) d'ici à la fermeture du parc français. Enfin, une série d'investissements en énergie renouvelable (2 500 à $8000 \mathrm{MW}$ d'ici 2007) est prévue par le décret de 2003 du gouvernement français, auxquels s'ajoutera entre 2010 et 2015 un prototype de nouvelle centrale nucléaire EPR de 1600 MW (annoncé après la parution du décret de mars 2003).

Dans ces circonstances, les grands investissements en France ne commenceraient qu'avec la fermeture du parc actuel de centrales nucléaires, donc après 2015. Dans le scénario d'une durée de vie de quarante ans pour les centrales nucléaires, EDF fermerait près de 50 GW entre 2017 et 2027 [12]. Mais ce scénario est de moins en moins certain. Beaucoup d'industriels du nucléaire soutiennent qu'une durée de vie de 50 années est accessible au prix de quelques investissements secondaires et à condition, bien sûr, que des organes irremplaçables comme les cuves satisfassent toujours aux critères de sécurité. Hors tout scénario de catastrophe, il existe donc une probabilité forte de voir reporter d'une décennie la date de démarrage de la fermeture du parc nucléaire français : 2027 au lieu de 2017.

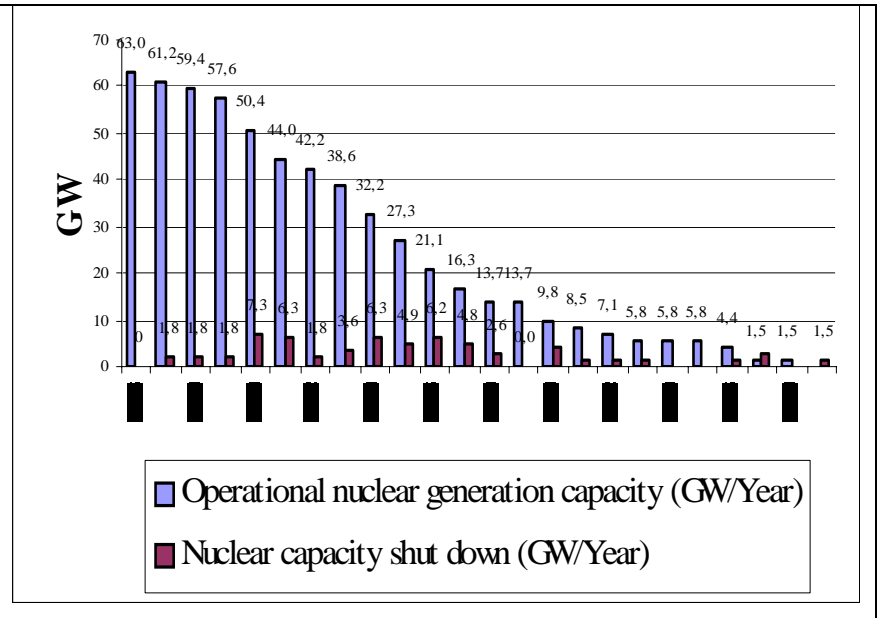

Fig. 3. Evolution «naturelle » du parc nucléaire français entre 2016 et 2039, sur la base d'une durée de vie de 40 ans (en GW).

Une seconde série d'incertitudes porte sur les modalités pratiques de ce futur réinvestissement massif. Remplacera-ton chacun des 63 GW de nucléaire existant aujourd'hui par du nucléaire nouveau? Sachant que la consommation française moyenne en 2020 serait de l'ordre de $550 \mathrm{TWH}$, avec une pointe hivernale moyenne inférieure à $90 \mathrm{GW}$ et un taux de croissance annuelle nettement inférieur à $1 \%$ ? D’autre part, si EDF conservait les mêmes niveaux de vente à l'étranger (70 à 80 TWh par an), les capacités de production correspondantes seraient-elles construites en France ou à l'étranger ? Dans le nucléaire ou en thermique classique ? Cela représente au moins $10 \mathrm{GW}$ de nucléaire en plus ou en moins. Enfin, cette période d'investissement massif ouvrira-t-elle une fenêtre d'entrée pour de nouveaux opérateurs, comme le dash for gas en Angleterre ? Ou faut-il anticiper dès maintenant que les investissements productifs futurs seront opérés essentiellement en intégration verticale, donc par les fournisseurs déjà en place sur le marché français ?

Si ce scénario d'investisseurs en intégration verticale devait se réaliser, on ne voit plus qu'une poignée d'investisseurs étrangers possibles. Electrabel, qui vise déjà $10 \%$ du marché français (soit 50 TWh à long terme); Endesa, si elle pouvait atteindre entre la moitié et le quart de l'objectif d'Electrabel (de 12 à $25 \mathrm{TWh}$ ); plus Enel qui devrait entrer en France sur invitation d'EDF et en contrepartie de la présence d'EDF en Italie (avec notamment le partage de centrales nucléaires en France).

Mais en fait, la plus grosse inconnue de l'investissement en intégration verticale n'est pas d'origine étrangère mais française. Il s'agit de Gaz de France. Le champion national du gaz est le principal concurrent potentiel d'EDF, tant sur le marché professionnel (avec 500000 consommateurs) que sur le marché domestique (avec 11 millions de consommateurs). C'est notamment sur le marché du Dual Fuel (fourniture conjointe de gaz et d'électricité) que Gaz de France possède des atouts concurrentiels difficiles à reproduire pour un nouvel entrant d'origine étrangère. Certes Gaz de France ne peut pas être l'égal de British Gas - Centrica, car l'usage du 
gaz est moins répandu en France qu'en Angleterre (moitié moins de consommateurs), et parce qu'EDF est une marque plus solide nationalement que celle de Gaz de France. Cependant, si Gaz de France n’était jamais fusionné avec EDF par le gouvernement français, on peut penser qu'il pourrait réaliser à terme au moins la moitié de la percée de British Gas - Centrica dans l'électricité, soit 10-12 \% du marché français. Additionné aux parts de marché des électriciens étrangers, ceci pourrait mettre un cinquième du marché français en dehors d'EDF et appeler environ $18 \mathrm{GW}$ de capacité en pointe. Mais comment tous ces nouveaux producteurs trouveront-ils en France tous les sites nécessaires pour implanter leurs dizaines de centrales, plus l'alimentation en combustible pour les faire fonctionner? Et à quelle date ? Sans attendre ces décennies futures, Gaz de France et Electrabel ont déjà commencé les préparatifs pour construire de nouvelles centrales CCGT en France.

\section{D'AUTRES MESURES D'ATTENUATION DU POUVOIR DE MARCHE DE L'ANCIEN MONOPOLE?}

Puisqu'on ne prévoit pas de reprise massive de l'investissement en France au cours de la prochaine décennie, et que les modalités pratiques de cette reprise demeurent très incertaines, qu'est-ce qui pourrait renforcer la frange concurrentielle dans le cas, très probable, où les axiomes de la politique française ne changeraient pas, et où le gouvernement français n'imposerait aucune restructuration industrielle ou désinvestissement en production / ventes aux champions nationaux [14], [15].

Sans doute des mesures additionnelles d'unbundling pourraient être prises pour le réseau de transport (transformable en une entreprise indépendante, possédée par un actionnaire public réellement neutre comme la Caisse des Dépôts), et pour les réseaux de distribution qui, n'appartenant pas en propre à EDF, pourraient être transformés en plusieurs sociétés publiques régionales (comme cela avait déjà été envisagé par la direction d'EDF à l'automne 2002). Dans la distribution, un unbundling régionalisé permettrait aussi un contrôle conjoint de la qualité de service et des performances économiques par les propriétaires des réseaux (les collectivités locales) et par le régulateur. Ces différentes mesures d'unbundling pourraient, en effet, faciliter l'action de la frange concurrentielle, mais sans réellement la renforcer.

Des modifications de la demande sont également envisageables. D'une part, le marché éligible s'est ouvert à tous les professionnels en juillet 2004 et sera élargi à tous les consommateurs domestiques en juillet 2007. D'autre part, toutes les entités publiques (ministères, municipalités, écoles et universités, hôpitaux ; etc.) finiront un jour par appliquer les règles de mise en concurrence pour les marchés publics de fourniture électrique. Ce qui pourrait créer un plus vaste espace d'action commerciale pour tous les fournisseurs qui souhaitent s'implanter en France. Il pourrait être envisageable, dans ce nouveau contexte, que se développent un ou deux «nouveaux entrants» (comme Poweo et Direct Energie tentent déjà de le faire) construisant des portefeuilles de 50000 à 100000 clients professionnels (2,5 à 5 TWh), et susceptibles de se revendre à terme comme "portails d'entrée » de plus gros opérateurs (étrangers comme Endesa ou Enel ; ou même français comme Gaz de France).

Du côté de l'offre, la Commission européenne pourrait-elle amplifier le programme de VPP qu'EDF a volontairement reconduit au-delà de la fin 2006 ? Il est aussi possible qu'EDF accepte une nouvelle mesure de ce genre comme preuve de bonne volonté "européenne », notamment si la réalisation d'un cadre de marché unifié n'a pas suffisamment avancé en Europe continentale. Une partie de ces VPP supplémentaires pourraient être assez peu formels et consister en accords bilatéraux, comme l'a été la mise à disposition de $1000 \mathrm{MW}$ de nucléaire d'EDF à Electrabel, ou comme les accords qu'EDF a discuté à plusieurs reprises avec ENEL.

Toujours en matière d'offre, les structures du marché intérieur français pourraient être protégées de toute nouvelle concentration par les opérateurs dominants. Ceci concerne tout particulièrement le marché du Dual Fuel (ou «biénergie », soit gaz + électricité), où aucun opérateur dominant dans une des deux énergies ne pourrait plus opérer de fusion acquisition avec un opérateur dominant dans l'autre énergie. Une autre variante, de type «italien », de cette nouvelle règle anti-trust serait d'établir un plafond de parts de marché pour le Dual Fuel : aucun opérateur ne pourrait détenir plus de (x) \% de ce nouveau marché tant qu'une structure suffisamment concurrentielle n’a pas émergé. La faisabilité de cette règle anti-trust pourrait être assurée ex ante par une filialisation régionalisée de l'activité des fournisseurs dominants. Ce qui permettrait des cessions ultérieures de parts de marché sans traumatisme industriel ni social. De telles cessions seraient d'ailleurs plus faciles à opérer entre EDF et Gaz de France qui sont tous deux des entreprises publiques et des champions nationaux français. D’autre part, toutes les activités de tous les acteurs dominants, que ce soit sur leur marché historique ou sur les nouveaux marchés, pourraient ête soumises à une surveillance permanente par une division spécialisée du régulateur, en liaison avec les autorités anti-trust françaises et européennes (ce qui suppose une évolution forte de l'attitude du gouvernement et du parlement français, qui pourrait survenir après la série d'élections programmées en 2007).

Enfin, quelques mécanismes de marché ou d'accès au marché pourraient être renforcés ou raffinés. Le mécanisme français de balancing pourrait être ouvert aux opérateurs de tous les pays frontaliers, comme le préparent le TSO et le régulateur français. Ce mécanisme pourrait aussi être transformé en véritable marché de court terme de l'énergie, adossé à des mécanismes concurrentiels d'allocation des capacités d'interconnexion coordonnés avec les autres TSOs sur toutes les frontières. La bourse Day Ahead de Powernext pourrait se coupler avec les bourses limitrophes (au Nord avec Belpex et APX, au Sud avec OMEL) et allouer directement des capacités d'interconnexion, comme plusieurs projets en discussion le suggèrent. Ces projets de couplage approfondi entre le marché français et les marchés voisins marquerait une 
étape importante de l'achèvement du marché intérieur de l'Union européenne et provoquerait, sans doute, une adaptation correspondante du marché allemand [15] et [16].

\section{CONCLUSION}

La France a engagé une réforme électrique particulière où le monopole public n’a été ni privatisé, ni détruit, ni déintégré. On y trouve néanmoins une frange concurrentielle, étrangère ou intérieure, entourant l'ancien monopole et bénéficiant du fait qu'il n’a pas abusé de son pouvoir de marché pour l'étouffer. Sur le front des prix de gros, les prix en France se tiennent au niveau de ceux de la production étrangère alternative qui est le thermique classique allemand. Les prix de vente aux grands clients éligibles ne traduisent pas, jusqu'en 2003, de comportement de maximisation des gains sur ce segment de marché, puisque ces prix se trouvaient souvent les plus bas d'Europe de l'Ouest. Les prix de vente aux clients domestiques non éligibles, fixés par le gouvernement français, ont comporté une marge apparente plus importante, quoi que toujours raisonnable comparativement aux pays voisins de la France.

Tous ces éléments correspondaient au profil d'un monopole public raisonnable, «collaborant" avec sa frange concurrentielle. Mais ils n'annoncent pas de future modification concurrentielle radicale des structures du marché français. Si cette modification radicale devait intervenir au moment du renouvellement du parc nucléaire français, il faudrait attendre encore longtemps. Car moins de $4000 \mathrm{MW}$ seront fermés avant 2029 si la durée de vie des centrales françaises passe à 50 ans.

En attendant ce très lointain futur, et en préparant l'ouverture du marché aux clients domestiques en 2007, une politique d'extension de la frange concurrentielle en France pourrait accroître les dispositifs d'electricity release. Bien que les VPP n'aient encore suscité l'apparition d'aucun opérateur alternatif d'envergure. Cette politique pourrait aussi, à la différence de l'Allemagne et de l'Espagne voisines, bloquer les fusions acquisitions entre les opérateurs français dominants du gaz et de l'électricité. Elle maintiendrait ainsi un potentiel de concurrence entre les deux champions nationaux français, notamment sur le marché du Dual Fuel. Ce potentiel serait renforcé par la privatisation partielle d'EDF et de GDF qui entretiendra leur rivalité latente, et par l'expansion lente d'Electrabel en France, accompagnée ou non par ENEL et Endesa (ou Iberdrola).

Par ailleurs, les mécanismes concurrentiels du marché français ou de l'accès au marché français pourraient encore être renforcés, notamment en les coordonnant ou en les couplant aux dispositifs concurrentiels des réseaux et des marchés voisins (Belgique, Pays-Bas, Espagne). Ce qui augmenterait d'autant l'ouverture et la transparence du marché français, ainsi que la crédibilité de la concurrence étrangère ou de quelques nouveaux entrants vis-à-vis du monopole national. Ces actions sur l'accès au marché français et sur les mécanismes de marché français contribueraient également à la construction d'un grand marché intérieur européen sur la plaque continentale.

Cependant la prolongation sur une ou deux décennies d'une politique française d' " encerclement concurrentiel » du monopole national pose un problème aigu de faisabilité. Cette politique cherche dans l'élargissement progressif des forces de marché influentes sur l'ancien monopole français les moyens de pérenniser le noyau dur de ses structures industrielles. La réalisation de cette politique demande donc une forte coopération des pays voisins et de la Commission européenne. Elle suppose ainsi des anticipations de gains de part et d'autre. Car elle ouvrirait les réseaux étrangers autant que le réseau français, et les marchés étrangers autant que le marché français, mais en gardant en place toutes les asymétries nationales de structure industrielle.

Une telle coopération multi pays et multi acteurs demeure possible dans le contexte actuel de forte remontée des prix sur l'ensemble de l'Union européenne et d'affaissement marqué de la compétitivité de la filière " Cycle Combiné au Gaz » (qui était le fer de lance des percées concurrentielles en GrandeBretagne et aux USA). En effet, ce contexte permet des multiples petites entrées simultanées "peu agressives » entre grands acteurs de la plaque européenne, au fil de la reprise spontanée de l'investissement, tout en opérant quelques très grosses opérations de taille européenne (OPA annoncées successivement sur Scottish Power ou SSE, sur Endesa, sur Electrabel, etc.). Les inconnues majeures des scénarios français concurrentiels de l'électricité s'étendent alors à l'évolution européenne de la filière gaz: l'accès au gaz combustible et au gaz fourniture en «Dual Fuel», et l'inachèvement de la consolidation entre électriciens et gaziers [17].

\section{REFERENCES}

[1] J-M Glachant, «The making of competitive electricity markets in Europe: no single way and no 'single market' » in Glachant J-M \& Finon D., Competition in European Electricity Markets. A Cross-country Comparison, Edward Elgar, 2003, pp. 7-40.

[2] D. Finon D., «Introducing competition in the French electricity supply industry » in Glachant J-M \& Finon D., Competition in European Electricity Markets. A Cross-country Comparison, Edward Elgar, 2003, pp. 257-284.

[3] EDF [Electricité de France], Rapports d'activité, Années 2002 à 2005.

[4] RTE [Réseau de Transport d'Electricité], Energie électrique en France, Années 2000 à 2005.

[5] CRE [Commission de Régulation de l'Energie], Rapports d'activité, Années 2002 à 2005.

[6] IAE [International Energy Agency], Energy Policies of IEA Countries, France 2004 Review, Juin 2004.

[7] Platts, European Electricity Review, June 2004.

[8] RTE [Réseau de Transport d'Electricité], Bilan prévisionnel 2006-2015, Juin 2004.

[9] J-M Glachant and M. Saguan, "An Institutional Frame to Compare Alternative Market Designs in EU Electricity Balancing," presented at American Economic Association, Boston, USA, January 2006.

[10] R. Belmans, J-M Glachant and L. Meeus, "Regional Electricity market Integation: France_Belgium-and The Netherlands”, KU Leuven and U. of Paris -Sud, September 2005. 
[11] M. Armstrong et A. Galli, "Are day-ahead prices for electricity converging in continental Europe? An exploratory data approach", CERNA; Ecole des Mines de Paris, février 2005.

[12] CEA [Commissariat à l'Energie Atomique] Nuclear power plants in the world, 2003.

[13] J-P Bouttes et J-M Trochet, "Marchés de gros et bourses de l'électricité en Europe et aux Etats-Unis: où en sommes nous?”, Conférence Jules Dupuit, Paris, 5 Décembre 2002.

[14] J-P Bouttes, "Sécurité d'approvisionnement et investissements dans l'électricité », Séminaire CFE-IFE, janvier 2005.

[15] J-M Glachant. and F. Lévêque, "Electricity Single Market in the European Union: What to do next?" Discussion paper of the European Union research project SESSA, Sept 2005, (http://www.sessa.eu.com/ documents/bruxellesp/SESSA_report_wp1.pdf). Working Paper CEEPR 2005-15 at MIT (web.mit.edu/ceepr). Main results presented at IDEI conference, Toulouse, May 2005.

[16] J-M Glachant, Y. Perez, V. Pignon et M. Saguan, « Un marché européen de l'électricité ou des marchés dans l'Europe? ", in Annales de l'Université de Paris I Panthéon-Sorbonne, à paraître, Avril 2006.

[17] Newberry, D. (2005). Refining Market Design, Discussion paper of the European Union research project SESSA, Sept 2005, (http://www.sessa.eu.com/documents/bruxellesp/

SESSA_report_wp3.pdf).

\section{BIOGRAPHIES}

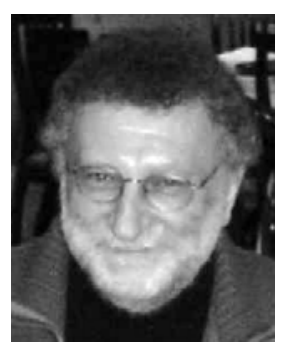

Jean-Michel Glachant est professeur d'Economie à l'Université Paris-Sud XI, Président de la Section d'Economie de la Faculté Jean Monnet, et responsable de l'équipe GRJM (Groupe Réseaux Jean Monnet) du laboratoire ADIS.

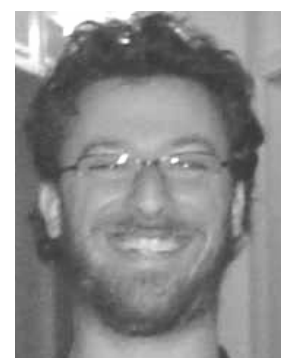

Marcelo Saguan possède un diplôme d'ingénieur de l'Université de Cuyo, Argentine et de l'Ecole Nationale d'Ingénieur de Metz, France. Il prépare une thèse d'économie à l'Université Paris-Sud XI (Faculté Jean Monnet) et à l'Ecole Supérieure d'Electricité (Supélec) dans le domaine du Market Design de l'électricité. 